\title{
Report on the Raster Capabilities of MIL-R-28002A and MIL-D-28003A
}

\section{Lynne S. Rosenthal}

U.S. DEPARTMENT OF COMMERCE Technology Administration National Institute of Standards and Technology

Computer Systems Laboratory Gaithersburg, MD 20899 


\title{
Report on the Raster Capabilities of MIL-R-28002A and MIL-D-28003A
}

\author{
Lynne S. Rosenthal
}

U.S. DEPARTMENT OF COMMERCE

Technology Administration

National Institute of Standards and Technology

Computer Systems Laboratory

Gaithersburg, MD 20899

November 1992

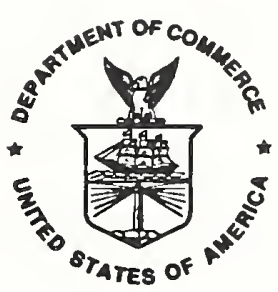

U.S. DEPARTMENT OF COMMERCE Barbara Hackman Franklin, Socretary

TECHNOLOGY ADMINISTRATION

Robert M. White, Under Secretary for Technolocy

NATIONAL INSTTUTE OF STANDARDS

AND TECHNOLOGY

John W. Lyons, Director 
Report on the Raster Capabilities of

MIL-R-28002A and MIL-D-28003A

Lynne S. Rosenthal

ABSTRACT

This report examines and compares the tiled raster graphics capabilities of military specifications MIL-R-28002A and MIL-D28003A. It presents reasons for which specification, MIL-R-28002A or MIL-D-28003A, to use to represent raster data.

Key words: CALS, CGM, raster graphics, tiled raster graphics, tiling 


\section{Acknowledgment}

The author would like to thank Frankie Spielman for sharing his expertise of MIL-R-28002A and for his constructive review of this document. 


\section{Table of Contents}

1. Introduction . . . . . . . . . . . . . . . . . 1

2. Acronyms and definitions . . . . . . . . . . . . . . 1

2.1 Acronyms used in this report . . . . . . . . . . . 1

2.2 Definitions used in this report . . . . . . . . . 2

3. Background . . . . . . . . . . . . . . . . . . . 3

3.1 Evolution of tiled raster in the CGM standard... . 3

3.2 Evolution of tiled raster standard and

MIL-R-28002 . . . . . . . . . . . . . . . . 4

4. Tiling . . . . . . . . . . . . . . . . . . . . . 5

4.1 Tile space representation . . . . . . . . . . . 6

4.2 CGM tile array . . . . . . . . . . . . . . . 7

4.3 MIL-D-28003A tile array requirements . . . . . . . 9

4.4 ODA raster tiling and NIST Raster DAP . . . . . . . 9

4.6 MIL-R-28002A requirements . . . . . . . . . . 10

5. Comparison of MIL-D-28003A and MIL-R-28002A tiling . . 10

5.1 Tile space . . . . . . . . . . . . . . . . 11

5.2 Relationship between raster content and the tile

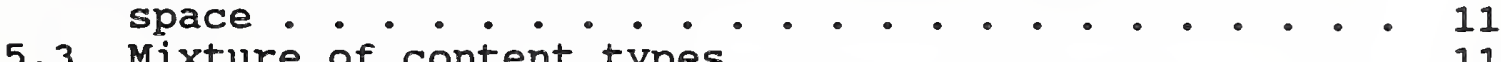

5.3 Mixture of content types . . . . . . . . . . 11

5.4 Picture versus document . . . . . . . . . . . . 11

5.5 Data stream encoding (interchange format) . . . . . 12

5.6 Group 4 compression ............... . . 12

5.7 Comparison of technical aspects . . . . . . . . 12

6. Choosing between the CALS standards . . . . . . . . . . 14

6.1 Document characteristics . . . . . . . . . . . 14

$6.2 \mathrm{Mix}$ of data................... . . 14

6.3 Color . . . . . . . . . . . . . . . . . 14

6.4 Compression method ............... . . 15

6.5 Raster storage and use ............ . . 15

6.6 SGML documents ................. . 15

6.7 Number and size of tiles . . . . . . . . . . . 16

6.8 Tile selection .................. 16

6.9 Encoding method ................ . 16

7. Availability of raster products . . . . . . . . . . 17

8. Conclusions and recommendations . . . . . . . . . . 17

9. References . . . . . . . . . . . . . . . . 19 


\section{Introduction}

The recently republished Computer Graphics Metafile (CGM) standard, ISO/IEC 8632:1992 [5], extends the capabilities of CGM to include additional graphics functionality. A tiled raster capability is among the new additions to ISO/IEC 8632:1992. MIL-D-28003A, Military Specification, Digital Representation for Communication of Illustration Data: CGM Application Profile [8], which is based on ISO/IEC 8632:1992, specifies the CGM elements necessary to implement tiled raster. As a result of this new raster capability, both MIL-D-28003A and MIL-R-28002A, Military Specification, Raster Graphics Representation in Binary Format [9], can represent raster graphics data in a digital, binary format.

The purpose of this report is to examine and compare the raster graphics capabilities of MIL-D-28003A and MIL-R-28002A, and to identify the requirements for when to use the tiled raster graphics capabilities of MIL-D-28003A (CGM) versus MIL-R-28002A. Only the tiled raster portion, i.e., Type II, of MIL-R-28002 will be addressed in this report.

The key to selecting the appropriate tiling method begins with an understanding of how each standard implements raster tiling. Thus, this report provides a general overview of each tiling method, followed by a comparison of the two methods. Based on this understanding, the advantages for using each method are presented. The report concludes with a discussion of the availability of raster tiling products and recommendations of which method to use.

\section{Acronyms and definitions}

\subsection{Acronyms used in this report}

Standards:

$\begin{array}{lll}\text { CALS AP } & \text { MIL-D-28003A } & \\ \text { CALS standards } & \text { MIL-R-28002A and MIL-D-28003A } & \\ \text { CGM:1987 } & \text { ISO/IEC 8632:1987 } \\ \text { CGM:1992 } & \text { ISO/IEC 8632:1992 } \\ \text { MIL-STD-1840 } & \text { Military Standard, Automated Interchange of } \\ & \text { Technical Information } \\ \text { ODA } & \text { ISO/IEC 8613:19891 }\end{array}$

${ }^{1}$ ISO/IEC 8613 will be republished in 1992 and will incorporate the addendum to ISO/IEC $8613 / 7$. 


$\begin{array}{ll}\text { ODA } / 7 & \text { Tiled Raster Graphics Addendum to ISO/IEC } \\ & 8613-7 \\ \text { SGML } & \text { ISO/IEC } 8879: 1986 \\ \text { TIFF } & \text { Tag Image File Format Specification }\end{array}$

Organizations:
ANSI
CITIS
American National Standards Institute
CCITT
Contractor's Integrated Technical Information Service
ISO
JCALS
International consultative committee for Telegraphy and Telephony
International organization for Standardization
OSI Joint Computer-aided Acquisition and Logistic Support
open Systems Interconnection

Abbreviations:

$\begin{array}{ll}\text { AP } & \text { Application Profile } \\ \text { ASN } & \text { Abstract Syntax Notation } \\ \text { CGM } & \text { Computer Graphics Metafile } \\ \text { DAP } & \text { Document Application Profile } \\ \text { Group } 3 & \text { CCITT Recommendation T4, Group } 3 \text { facsimile } \\ \text { Group } 4 & \text { CCITT Recommendation T6, Group } 4 \text { facsimile } \\ \text { OIW } & \text { Open Systems Environment Implementors' } \\ & \text { Workshop } \\ \text { Type I } & \text { Untiled raster graphics data } \\ \text { Type II } & \text { Tiled/untiled raster graphics data }\end{array}$

\subsection{Definitions used in this report}

When dealing with different standards, it is not unusual to see different terms representing the same or similar concepts. The CGM and ODA standards are no exception. The following are some of the equivalent terms used in this report.
$\underline{\text { CGM }}$
$\underline{O D A}$
cell
tile array
CGM elements
pel
pel array ${ }^{2}$
ODA constitutents and attributes

\footnotetext{
${ }^{2}$ The definition of pel array pertains to both tiled and untiled raster images. Since this report only addresses tiling, the meaning of pel array will be limited to tiled raster images and thus equivalent to tile array.
} 
In general, the CGM terminology will be used throughout the report, with ODA terminology in parenthesis. However, ODA terminology will be used when describing ODA-related concepts.

The definitions given in the CGM and ODA standards are applicable to this report. In addition, the following definitions apply for the terminology as it is used in this document:

conforming metafile: A metafile that complies with the specifications in MIL-D-28003A.

content: Information that is intended for human perception, e.g., text, raster graphics, geometric or vector graphics.

metafile: a collection of CGM elements.

ODA raster document: An ODA document that contains only the raster images (i.e., excludes text and geometric graphics).

raster content information: See tile array.

tile: A rectangular region of a raster image.

tile array: A sequence of one or more two-dimensional, equally sized individual tiles which all together form a contiguous rectangular block of tiles. The ODA terms pel array and raster content information are synonymous with tile array.

\section{Background}

\subsection{Evolution of tiled raster in the CGM standard}

CGM became an American National Standard in 1986 and a Federal Information Processing Standard and International Standard in 1987. CGM specifies an interchange format suitable for storage and transfer of picture description information. Although the picture description information is typically vector data, it may be raster data.

Until CGM:1992, raster data could be represented in CGM using the CELL ARRAY primitive. The standard allowed for compression of the raster as well as color. However, practical experience has shown that the CELL ARRAY primitive was not the most efficient method for handling raster data and was too limited to meet CALS requirements [7]. In general, a CGM using CELL ARRAY to represent raster data is large, cumbersome, and slow to process and display. Moreover, compression techniques are limited and do not include common techniques such as Group 3 or Group 4 facsimile. 
The need to accommodate the use of raster data is a fundamental CALS requirement [2]. This requirement was one of several CALS requirements which propelled the ANSI effort to. enrich CGM functionality in support of technical illustration and publishing applications.

In 1988, work began on Amendment 3 to CGM. This work comprised of a set of new elements which would extend the capabilities of the CGM:1987 to support advanced 2D drawing capabilities, improved text and font support, and additional raster graphics capabilities. A complete list of functional areas addressed by Amendment 3 can be obtained in the CGM Addendum 3 new work item proposal [1].

In 1991, Amendment 3 was completed and resulted in more than fifty new CGM elements. Among the new elements in Amendment 3 , are the Tile Array elements which describe raster graphics data. CGM raster was specifically designed to be functionally equivalent to TIFF, ODA raster, and MIL-R-28002A.

Rather than publish Amendment 3 as a separate document, the CGM standard was revised to contain Amendment 3 along with Amendment 1 and a large number of resolved defect reports. The resulting standard, CGM:1992, replaces CGM:1987 and defines three versions. Version 1 corresponds to the original standard, CGM:1987, version 2 corresponds to Amendment 1, and version 3 corresponds to Amendment 3 .

Based on CGM:1992, MIL-D-28003A describes the allowable elements and parameters which may be contained in a CGM and specifies implementation requirements for generators and interpreters. MIL$\mathrm{D}-28003 \mathrm{~A}$ also defines three versions, such that each version is a subset of the corresponding version of CGM:1992. The tiled raster capability is supported in version 3. Thus, only metafiles which conform to version 3 of MIL-D-28003A can contain the Tile Array elements.

\subsection{Evolution of tiled raster standard and MII-R-28002}

In 1987, responding to CALS raster requirements, an ad-hoc group of industry and government raster graphics experts began developing a method for tiling large engineering drawings. The resulting tiled raster specification was incorporated into the open Document Architecture and Interchange Format (ODA) standard, raster content architecture (ISO $8613 / 7$ ). ODA was chosen to accommodate this raster work since it was the only international standard that dealt with raster graphics. Moreover, the compression algorithms used in the ODA raster content architecture (i.e., CCITT Group 3 and Group 4) were the same algorithms that were specified by the tiled raster group. 
ODA specifies rules for describing the logical and layout structures of documents and for specifying the character, raster, and geometric content of documents. Moreover, ODA defines the format to be used for the interchange of ODA documents. ODA offers a wide range of choices of different sets of features and information. As a result, functional profiles have been developed that specify subsets of ODA that are appropriate for particular applications. These subsets of ODA are called document application profiles (DAPs).

A DAP pertaining to ODA documents containing only raster data was developed by the ODA special Interest Group of the NIST Open Systems Interconnection (OSI) Implementors Workshop ${ }^{3}$ [13]. The DAP is based on ODA as defined in ISO 8613 and the Tiled Raster Graphics Addendum to ISO 8613, Part 7. The DAP, referred to as the NIST Raster DAP, allows large format raster documents to be interchanged between equipment designed for raster processing. Such documents contain bitonal raster graphics data, such as engineering drawings and illustrations.

MIL-R-28002A defines the structure and encoding of raster data files. Type II of MIL-R-28002A is a delimitation of the NIST Raster DAP to suit the CALS requirements. Type II raster graphics requirements can be used for tiled or a mixture of tiled and untiled raster graphics data.

The reader should note that $M I L-R-28002 \mathrm{~A}$ is currently being revised. The revision, MIL-R-28002B, will be published in late 1992 and will contain a new DAP, titled ODA Raster DAP. However, changes to MIL-R-28002B do not invalidate this report and will be noted herein where appropriate.

\section{Tiling}

Tiling is a method for subdividing a large raster image into smaller, nonoverlapping rectangular regions called tiles. It provides an efficient and flexible method for handling raster data.

Tiling is best suited for large format drawings or illustrations typically associated with engineering design. The subdivision of a drawing into tiles permits the use of only those portions of an image required at a given time by the application. This can result in reduced requirements for workstation memory and workstation display area [9].

${ }^{3}$ The name of this workshop has changed to: Open systems Environment Implementors' Workshop. 
Each tile can be encoded as uncompressed or compressed tiles according to a selected compression method. The capability to encode each tile separately, using different: compression algorithms, maximizes the possible compression of the entire tiled raster image. In addition, tiling enables graphics and/or raster systems to perform the compression and decompression activities in parallel upon the drawing tiles [9].

\subsection{Tile space representation}

The basic concepts for tiling are the same in CGM:1992 and ODA raster. Both represent the tile space as a tile array (pel array) and a set of tiles as shown in figure 1. The tile array in figure 1 contains twenty tiles.

The tile array consists of one or more equally sized, nonoverlapping tiles. The tiles are ordered sequentially along the cell (pel) direction and then in the line progression direction as illustrated in figure 1.

Although CGM tiling and ODA raster tiling are similar and use the same general model, the mechanism for implementing tiling is different. An overview of the CGM and ODA raster specifications is provided in the following sections. 


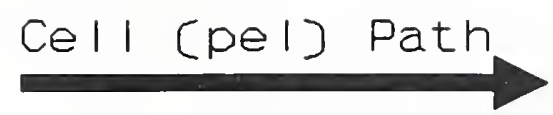

\begin{tabular}{|c|c|c|c|c|}
\hline & 1 & 2 & 3 & 4 \\
\hline Line & 5 & 6 & 7 & 8 \\
\hline 5 & 9 & 10 & 11 & 12 \\
\hline 13 & 14 & 15 & 16 \\
\hline 17 & 18 & 19 & 20 \\
\hline
\end{tabular}

Figure 1. Example of the tile array content and ordering.

\subsection{CGM tile array}

The CGM tile array can define either bitonal or color raster data, in either an uncompressed or compressed form. Two classes of CGM elements and four CGM elements are used to specify the tile array and its raster contents:

\section{delimiter elements: $\quad$ BEGIN TILE ARRAY \\ END TILE ARRAY}

graphical primitive elements: TILE

BITONAL TILE

The tile array is defined and delimited by the CGM elements BEGIN TILE ARRAY and END TILE ARRAY. The complete tile array is formed by 
one or more tiles between the BEGIN TILE ARRAY and END TILE ARRAY. ${ }^{4}$ The general structure of metafile containing a $2 \times 2$ tile array is as follows:

\author{
BEGIN METAFILE \\ METAFILE VERSION \\ METAFILE DESCRIPTION \\ BEGIN PICTURE \\ BEGIN PICTURE BODY \\ BEGIN TILE ARRAY \\ TILE or BITONAL TILE \\ TILE or BITONAL TILE \\ TILE or BITONAL TILE \\ TILE or BITONAL TILE \\ END TILE ARRAY \\ END PICTURE \\ END METAFILE
}

The tile array elements are embedded within the structure of a metafile. In addition to these elements, a metafile must contain several required elements and may contain other visual components of the picture (e.g., text, boxes, and lines). In general, the overhead, in terms of file size and processing, of a metafile consisting of the required elements and a tile array is minimal.

The BEGIN TILE ARRAY element contains the parameters which apply to the collection of tiles which make up the tile array. These parameters contain the positioning and dimensioning information and determine the number of tiles in the tile array.

The tiles which comprise the tile array are defined by either the TILE or BITONAL TILE element. The TILE element is used to represent color raster, whereas the BITONAL TILE represents raster images with only two colors. Each element (i.e., TILE or BITONAL TILE) contains only the raster content of a single raster tile, its compression method, a row padding indicator, and the color specifiers. The order of the data (i.e., bit ordering) is in the "down" direction, or most significant bit (MSB) to least significant bit (LSB). A detailed description of these elements and parameters can be found in CGM:1992.

${ }^{4} \mathrm{~A}$ tile array containing one tile is how an untiled image is represented in CGM. 


\subsection{MIL-D-28003A tile array requirements}

Both the TILE and BITONAL TILE elements are allowed in metafiles conforming to MIL-D-28003A. Tiles may be compressed using any one of the six compression methods. Each tile shall be no larger than one $1024 \times 1024$ image and no more than 256 tiles.

\subsection{ODA raster tiling and NIST Raster DAP}

ODA raster tiling defines bitonal raster data in either an uncompressed or compressed form. Additionally, the ODA raster tiling mechanism contains information about the document containing the raster data as well as where the raster data fits in the document.

The tiling is defined in ODA/7, with the NIST Raster DAP defining a specific subset of ODA raster. The DAP describes the features of ODA that are needed to support the interchange of documents containing only raster graphics content [13]. These documents will be referred to as ODA raster documents.

An ODA raster document consists of the following sequence of ODA data items: document profile, presentation style, document layout description, and content portion description. Each of these data items or constitutents contains the attributes which describe the document and/or raster data. The general structure of an ODA raster document ${ }^{5}$ is shown in figure 2. A detailed description of each constituent is contained in the NIST Raster DAP [13] and the ODA standard [4].

The document profile, document layout structure, and basic page(s) contain information which apply to the document as a whole or to the placement of the raster image within the document and on the page. Each basic page contains a tile index whose elements match the order of the tiles. The presentation styles guide the format and appearance of the raster content. In particular, the style contains a set of attributes which contain the positioning and layout information of the raster content.

The content portion is the actual raster data and its associated attributes. These attributes contain information on the compression (encoding) method, the tile array size, and the tiling offset. Additionally, the NIST Raster DAP specifies that the size of each tile within the tile array is always a $512 \times 512$ square.

5In MIL-R-28002B, the general structure of an ODA raster document will be slightly different. 
Document Profile

Presentation Styles $\begin{gathered}\text { (optional } \\ \text { constitutent) }\end{gathered}$

Document Layout Root

Repeat as necessary

Bas ic Page Layout Object

Content Portion

Figure 2. structure of an ODA raster document

\subsection{MIL-R-28002A requirements}

MIL-R-28002A, Type II data is a MIL-STD-1840 header wrapped around an ODA-style document as specified in the NIST Raster DAP [11]. Some of the features of the NIST Raster DAP are further restricted by requirement statements in MIL-R-28002A. In particular, MIL-R$28002 \mathrm{~A}$ respecifies the default pel spacing for large-format engineering drawings, requires the image size to be a multiple of eight, and designates the bit ordering to be most significant bit to least significant bit.

\section{Comparison of ML-D-28003A and MIL-R-28002A tiling}

There is great similarity between the tiling mechanisms in MIL-D$28003 \mathrm{~A}$ and MIL-R-28002A. This is not unexpected, since the tiling mechanism in MIL-D-28003A is based on the Tiled Raster Interchange format that has been developed for ODA/7. Since MIL-R-28002A and MIL-D-28003A are based on the ODA and CGM standard, many of the similarities and differences stem from these two standards. These similarities and differences are discussed below. 


\subsection{Tile space}

As discussed in section 4.1 , the model for describing the tile space is the same for both CALS standards. Both standards structure and describe the tile space hierarchically as a tile array containing one or more tiles. The tile array specifications pertain to the entire tile space (i.e., every tile in the tile array), and each tile has its own set of specifications.

The ODA tile space differs from the CGM model, in that it also defines document-related specifications. These specifications describe how the tile space fits into the document. Since CGM has no concept of a "document," there are no equivalent specifications.

\subsection{Relationship between raster content and the tile space}

The relationship of the raster content to the tile space, including the ordering and layout, is the same for both standards (see section 4.1). Additionally, both standards allow for the actual raster image to be smaller than or "offset from" the whole tile array. When an offset is specified, a border of unused cells surrounds the actual image.

\subsection{Mixture of content types}

The NIST raster DAP and MIL-R-28002A specify that Type II data file can contain only raster content. The other ODA content types (i.e., text and geometric graphics) are not allowed in Type II data file. CGM, on the other hand, can contain text and vector graphics content. The raster data constitutes just one of the many visual components that may be contained in a CGM picture.

\subsection{Picture versus document}

Although both CGM and ODA are interchange formats, the purpose of CGM is to describe pictures, whereas the purpose of ODA is to describe documents. This difference in purpose is a major difference between the raster implementation in the two standards.

In the CGM standard, the metafile contains no information about how the picture will be used in an application or how the picture is contained in a document. Moreover, the raster capability in CGM is confined to a single picture. The tile array elements have no effect on other drawing actions, nor on other pictures in the metafile. 
In the ODA standard, information about the document and its content (e.g., raster) is the essence of ODA. In fact, ODA/7 defines the raster capability and its relationship to the ODA document.

\subsection{Data stream encoding (interchange format)}

CGM uses a binary data format to represent a picture conforming to MIL-D-28003A. For each CGM element, an explicit representation in terms of bits, octets, and words is specified.

ODA uses the office document interchange format (ODIF) to represent documents containing raster data. ODIF is an abstract data syntax in which the constituents and attributes are represented by a hierarchy of data structures and data items specified using the abstract syntax notation ASN.1 [4].

\subsection{Group 4 compression}

MIL-R-28002A restricts the compression of Type II raster graphics to Group 4 compression. Group 3 compression is specifically not supported. ${ }^{6}$ MIL-D-28003A allows both Group 4 and Group 3 compression.

\subsection{Comparison of technical aspects}

The following table presents a comparison of the technical aspects and elements that define the tile array and tile. It is interesting to note that the terminology may be different, but most of the functionality and values of elements are the same. If the terminology is different, the CGM term is given first, followed by the ODA term. Actual CGM element and ODA attribute names are shown in italics.

${ }^{6}$ Both Group 3 and Group 4 are allowed in the NIST Raster DAP. The restriction to Group 4 is a MIL-R-28002A requirement. 


\section{COMPARISON OF TECHNICAL APSECTS}

\begin{tabular}{|c|c|c|c|}
\hline TECHNICAL ASPECT & $\begin{array}{l}\text { MIL-R-28002A } \\
\text { VALUES }\end{array}$ & $\begin{array}{l}\text { MIL-D-28003A } \\
\text { VALUES }\end{array}$ & Comments \\
\hline Bit ordering & MSB to LSB & MSB to LSB & \\
\hline Partical tiles & $\begin{array}{l}\text { recommend that } \\
\text { unimaged pels set } \\
\text { to background }\end{array}$ & $\begin{array}{l}\text { recommend that } \\
\text { unimaged cells set to } \\
\text { background }\end{array}$ & \\
\hline Padding/byte boundaries & $\begin{array}{l}\text { pad line dimensions } \\
\text { to mulitple of } 8\end{array}$ & $\begin{array}{l}\text { no requirement, but } \\
\text { has a row padding } \\
\text { indicator }\end{array}$ & $\begin{array}{l}\text { CGM: indicator } \\
\text { gives the row } \\
\text { padding, if any }\end{array}$ \\
\hline Clipping & yes & yes & \\
\hline $\begin{array}{l}\text { Orientation } \\
\text { cell (pel) path } \\
\text { line progression }\end{array}$ & $\begin{array}{l}0,90,180,270 \\
90,270\end{array}$ & $\begin{array}{l}0,90,180,270 \\
90,270\end{array}$ & \\
\hline Tile offset & $\begin{array}{l}2 \text { non-negative } \\
\text { integers }<=512\end{array}$ & $\begin{array}{l}2 \text { non-negative } \\
\text { integers }\end{array}$ & \\
\hline Compression method & $\begin{array}{l}\text { null background } \\
\text { null foreground } \\
\text { T6 } \\
\text { bitmap }\end{array}$ & $\begin{array}{l}\text { null background } \\
\text { null foreground } \\
\text { T6 } \\
\text { T4 } 1 \text { dimensional } \\
\text { T4 } 2 \text { dimensional } \\
\text { bitmap } \\
\text { run length }\end{array}$ & \\
\hline Maximum number of tiles & & 256 & \\
\hline $\begin{array}{l}\text { cell size in path and line direction } \\
\text { pel spacing }\end{array}$ & 200,300 pels/inch & any positive value & \\
\hline $\begin{array}{l}\text { \# cells in path direction } \\
\text { \# pels per line }\end{array}$ & any positive value & any positive value & \\
\hline $\begin{array}{l}\text { \# cells in line direction } \\
\text { \# lines }\end{array}$ & any positive value & any positive value & \\
\hline $\begin{array}{l}\text { \# cells/tile in path direction } \\
\text { \# pel/tile/line }\end{array}$ & 512 & 1024 & $\begin{array}{l}\text { CGM: value } \\
\text { corresponds to }\end{array}$ \\
\hline $\begin{array}{l}\text { \# cell/tile in line direction } \\
\text { \# lines/tile }\end{array}$ & 512 & 1024 & $\begin{array}{l}1048576 \text { compressed } \\
\text { color specifiers }\end{array}$ \\
\hline
\end{tabular}




\section{Choosing between the CALS standards}

This section presents the reasons for choosing which CALS standard, MIL-R-28002A or MIL-D-28003A, to use to represent raster data.

\subsection{Document characteristics}

If the intent of representing the raster data is to include information about the document which will contain the raster image, then MIL-R-28002A should be chosen. MIL-R-28002A includes ODA document structuring and presentation information, such as page dimensions, multiple pages, and page position.

For collections of raster images, where the intent is to represent the raster data and not be concerned with the document or application which will ultimately contain the raster image, then MIL-D-28003A is the viable choice. The CGM raster representation is simply the raster image without the overhead of the ODA structure.

\subsection{Mix of data}

If the raster will be enhanced and/or mixed with other types of data, e.g., text and vector graphics, then MIL-D-28003A should be chosen. Other types of data such as pointers, lines, or text may be added to a raster image for purposes of annotation, labeling, or highlighting.

As a raster-only representation, MIL-R-28002A cannot represent raster images which contain text and/or graphics data. This limitation to raster-only is not an ODA limitation. There is an OIW DAP [12] that will allow text and graphics to be combined with the raster image.

\subsection{Color}

Both MIL-R-28002A and MIL-D-28003A can represent bitonal raster data. However, only MIL-D-28003A can represent color raster data. 


\subsection{Compression method}

MIL-D-28003A specifies the same compression methods as MIL-R-28002A plus two additional methods, Group 3 and run length.

If Group 3 compression/decompression is required, then MIL-D-28003A should be chosen. Using MIL-D-28003A raster, an image can be encoded according to the one or two dimensional scheme defined by CCITT Recommendation T.4. The T.4 (denoted T4 in CGM:1992) compression is useful since Group 3 facsimile is the predominate method used by facsimile machines today.

Alternatively, MIL-D-28003A raster can be encoded using the run length compression method. Run length compression is an effective way to compress images where there is high repetition. For example, if the compression type is "run length," then a sequence of color cells with identical values is replaced by a count and the color.

\subsection{Raster storage and use}

If the intent is long-term storage of raster data, then either CALS standard is appropriate. However, long term storage is an explicit objective of MIL-R-28002A and the reason for using Group 4 compression.

If the raster image will be integrated into other software applications, such as publishing systems and drawing packages, then either CALS standard is appropriate. However, MIL-D-28003A might be the better choice because it represents the raster image without the extra document structure specifications. For most software applications, only the raster image is imported; any document information would most likely be discarded. Moreover, CGM software $^{7}$ is available in a wide range of applications including presentation graphics, word processing, publishing, illustration, CAD, mapping, graphics programming (e.g., GKS and PHIGS), statistical analysis, project management, and even ODA.

\subsection{SGML documents}

The standard Generalized Markup Language (SGML) is a method for describing the content and structural elements of an electronic document. An SGML document can include, via reference, CGM pictures

\footnotetext{
${ }^{7}$ Current CGM software corresponds to the functionality in CGM:1992 version 1. Since the CGM raster capability is a new function in CGM:1992 version 3, there are no current implementations. We believe this new functionality will be incorporated into CGM software in the near future.
} 
as well as ODA raster images. Since SGML has its own document structure information, the ODA document information contained in a MIL-R-28002A Type II raster image is unessential and irrelevant to the SGML document. As a picture representation, MIL-D-28003A raster would be well suited for use in SGML documents.

\subsection{Number and size of tiles}

MIL-D-28003A limits the number of tiles to 256 and the size of each tile to $1024 \times 1024$ cells. MIL-R-28002A has no limit on the number of tiles, but does constrain the tile size to $512 \times 512$ pels. Thus, if the number of tiles is greater than 256, MIL-R-28002A should be used. Similarly, if the size of the tiles is larger than $512 \times 512$ pels, MIL-D-28003A should be used.

In general, if the image is very large and the actual number of tiles are unknown but likely to be greater than 256, then MIL-R$28002 \mathrm{~A}$ should be used. Moreover, it may not matter that the size of each tile is limited. The raster image can still be represented by MIL-R-28002A. It may take more tiles to represent the image, but this is not a problem since Type II raster can contain any number of tiles.

\subsection{Tile selection}

MIL-R-28002A Type II raster includes a tile index parameter which can be used to identify specific tile locations within the tile array. Thus, if a specific tile is of interest, it can be selected from the tile array. MIL-D-28003A, and more generally CGM:1992, does not have this capability.

\subsection{Encoding method}

The encoding method should not be a major factor in choosing between the two standards. The encoding, whether it be ASN.1 or binary, is transparent to the user. Both methods are efficient ways of encoding data for interchange, and each method has its community of support. ASN.1 is the preferred method for interchange in the OSI and networking communities. The binary encoding is the preferred method of CGM vendors in the United states. 


\section{Availability of raster products}

The key to choosing which CALS standard to use hinges on the availability of products which contain the raster capability. Currently and unfortunately, neither MIL-R-28002A Type II raster products nor MIL-D-28003A raster products are available. However, we know of several Type II raster products which will soon appear in the marketplace.

The MIL-R-28002A products being developed will be available on a variety of platforms including Unix-based workstations and personal computers. For many of these products, the raster encoder/decoder is one of several supported graphics formats. Not surprisingly, products which support multiple graphics formats and can translate between formats are called graphics translation software.

The raster capability specified in MIL-D-28003A is one of several new capabilities added to the CGM standard. Because CGM:1992 is a new standard, there are no implementations supporting the new functionality as specified in the standard. Given the wide acceptance and use of CGM today, ${ }^{8}$ we are confident that vendors will upgrade their products to support the new functionality in CGM:1992. Already, we know of several vendors who are working on CGM products which will support tiled raster. These products should appear in the marketplace within the next year.

\section{Conclusions and recommendations}

The raster capability in MIL-R-28002A and MIL-D-28003A provides for the digital representation of tiled raster graphics data. This representation of raster graphics data enables the government to acquire raster graphics data which can be electronically accessed, stored, or interchanged. Representing raster in digital format is consistent with the objectives and use of data in other Department of Defense programs such as JCALS and CITIS.

The tiled raster capability specified in MIL-D-28003A is functionally equivalent to the tile capability in MIL-R-28002A. Because of their similarity, either CALS standard could be used to represent bitonal uncompressed (bitstream) raster data or bitonal compressed (Group 4) raster data.

The major differences between the raster representations in the two CALS standards are due to the underlying ODA document architecture

\footnotetext{
${ }^{8}$ There are over 350 implementations of CGM on all types of platforms.
} 
in MIL-R-28002A and the additional functionality of CGM raster in MIL-D-28003A.

MIL-R-28002A Type II specifies a simple ODA raster graphics document. As such, information about the document containing the raster data is included in the digital representation. Moreover, MIL-R-28002A supports only raster graphics content that is a bitonal black and white image. Other contents, such as text and vector graphics, are not supported by MIL-R-28002A.

On the other hand, a tiled raster specified by MIL-D-28003A does not contain any document information. In CGM, only the raster data is represented. The raster data may be the entire CGM picture or constitute one of several visual components of a picture. Thus, a CGM with raster data may contain annotation (i.e., text), pointers, lines, etc.

The additional functionality of the CGM tiled raster is its ability to represent color raster images and its support for Group 3 compression. MIL-D-28003A supports these capabilities.

Choosing between the two CALS standards is a matter of capability, preference, and availability.

We recommend that $M I L-D-28003 \mathrm{~A}$ be used to represent raster data if the capabilities of CGM raster (i.e., color, Group 3 compression, and mix of data) are required. Additionally, if working in a CGM environment is desirable because the raster image will be used in conjuction with other application software, then MIL-D-28003A is a good choice.

We recommend that $M I L-R-28002 \mathrm{~A}$ be used if document structuring information is important to the raster representation and long term storage of raster data is required. Additionally, if the number of tiles and tile size exceeds 256 and $1024 \times 1024$, respectively, then MIL-R-28002A should be used. We also recommend that MIL-R$28002 \mathrm{~A}$ be used if the raster image will be used with raster-based software products (e.g., paint programs).

Ultimately, the choice comes down to, what raster products are available? Currently neither MIL-R-28002A nor MIL-D-28003A products are readily available. However, products which implement MIL-R-28002A Type II raster will probably be available sooner than products which implement MIL-D-28003A raster. Thus, if you need a raster product today, the choice obviously is to look for a MIL-R28002 A product.

Once products which implement either CALS standard are available, their usability and faithfulness to the standard will need to be judged. 


\section{References}

[1] CGM Addendum 3 New Work Item Proposal, ANSI X3H3/89-48

[2] DOD/NBS/Industry Workshop on Automated Technical Manual Systems and Automated Data Repositories, held at NBS, June $24-$ $25,1986$.

[3] Federal Information Processing Standards Publication (FIPS), Computer Graphics Metafile (CGM), FIPS PUB 128, 16 March 1987.

[4] ISO/IEC 8613:1991, Information Processing - Text and office Systems: Open Document Architecture (ODA) and Interchange Format.

[5] ISO/IEC 8632:1992, Information Processing Systems - Computer Graphics - Metafile for the storage and Transfer of Picture Description Information.

[6] ISO/IEC 8879:1992, Information processing - Text and office systems - standard Generalized Markup Language (SGML).

[7] Kemmerer, Sharon J., CGM Registration for CALS Requirements $A$ Technical study Completed for the Computer-aided Acquisition and Logistic support (CALS) Program Fiscal Year 1987. NBSIR 88-3728, March 1988 .

[8] Military Specification, Digital Representation for Communication of Illustration Data: CGM Application Profile, MIL-D-28003A, 15 November 1991.

[9] Military Specification, Raster Graphics Representation in Binary Format, Requirements for, MIL-R-28002A, 30 November 1990.

[10] Military standard, Automated Interchange of Technical Information, MIL-STD-1840A, 22 December 1987.

[11] Spielman, Frankie E., and Louis H. Sharpe II, Tiled Raster Graphics and MIL-R-28002A: A Tutorial and Implementation Guide, NISTIR 4567, April 1991.

[12] Stable Implementation Agreements for open systems Interconnection Protocols, Part 22, ODA Image DAP, NIST Special Publication 500-202, Version 5, Edition 1, 1991.

[13] Stable Implementation Agreements for open systems Interconnection Protocols, part 23, ODA Image DAP, NIST Special Publication 500-202, Version 5, Edition 1, 1991. 
NIST-114A

(REV. 3-90)
U.S. DEPARTMENT OF COMMERCE NATIONAL INSTITUTE OF STANDARDS AND TECHNOLOGY

BIBLIOGRAPHIC DATA SHEET
1. PUBLCATION OR REPORT MUMBER

NISTIR 4970

2. PERFORMING ORGANIZATION REPORT MUMBER

3. PURUCATION DATE

...... NOV EMBER . 1992

\section{TITLE AND SUBTITLE}

Report on the Raster Capabilities of MIL-R-28002A and MIL-D-28003A.

5. AUTHOR(S)

Lynne S. Rosenthal

6. PERFORMING OAGANIZATION (IF JOINT OR OTHER THAN MIST, SEE INSTAUCTIONS)

U.S. DEPARTMENT OF COMMERCE

MATIONAL INSTITUTE OF STANDARDS AND TECHNOLOQY

CANTHERSBURG, MD 20890

7. CONTRACT/ORANT NUMBEA

8. TYPE OF REPORT AND PEAIOD COVERED

9. SPONSOAING OACANIZATION MAME AND COMPLETE ADDRESS (STREET, CTT, STATE, ZIP)

10. SUPPLEMENTAAY MOTES

11. ABSTAACT (A 200-WORD OA LESS FACTUAL SUMMARY OF MOST SIGMIFICANT INFORMATION. IF DOCUMENT INCLUDES A SIONIFICANT BIBLIOQRAPHY OR UTERATURE SURVEY, MENTION IT HERE)

This report examines and compares the tile raster graphics capabilities of military specifications MIL-R-28002A and MIL-D-28003A. It presents reasons for which specification, MIL-R-28002A or MIL-D-28003A, to use to represent raster data.

12. KEY WORDS (6 TO 12 ENTAIES; NPHABETICAL ORDER; CAPTALIZE ONLY PROPER MAMES; AND SEPARATE KEY WORDS BY SEMICOLOMS) Computer-aided Acquisition and Logistic Support (CALS); Computer Graphics Metafile (CGM); raster graphics, tile raster graphics, tiling.

\section{AVALABIUTY}

$X X$ UNUMTED

FOR OFFICLAL DISTAIBUTIOH. DO NOT REEASE TO MATIOMAL TECHNICN INFORMATION SERVCE (NTIS).

ORDER FROM SUPERINTENDENT OF DOCUMENTS, U.S. GOVERMMENT PAINTIMO OFFCE, WASHINOTON, DC 20402.

ORDER FROM MATIONAL TECHMICAL INFORMATION SERVICE (NTIS), SPAINOFIELD, VA 22161.
14. NUMBER OF PRINTED PAOES

25

15. PAICE

$\mathrm{AO} 2$ 


